\title{
Evolución de los serotipos, fagotipos y resistencia a antimicrobianos de Salmonella sp en el departamento de salud 02 de la provincia de Castellón, España (2000-2006)
}

\author{
Ma Dolores Tirado B., Rosario Moreno M., Ma Elena Celades P., Juan Bellido-Blasco y Francisco J. Pardo S.
}

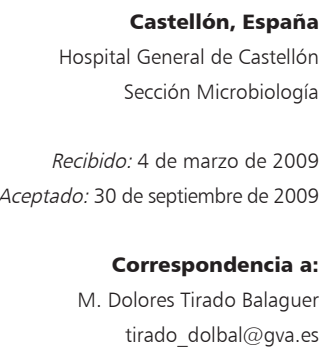

Dolores Tirado Balaguer

tirado_dolbal@gva.es

\section{Evolution of serotypes, phagotypes and antibiotic resistance of Salmonella spp in the 02 health district of Castellon, Spain (2000-2006)}

Retrospective study of serotypes, phage types and antibiotic resistance of Salmonella spp isolates in the 02 Health District of Castellon, Spain (2000-2006). Strains were serotyped using commercial sera, and they were tested for antimicrobial susceptibility with automated systems. Serotyping confirmation and phage typing were performed by the National Reference Laboratory. A total of 1505 strains were isolated, with 49 different serotypes, being the most frequent Enteritidis. The most common serotype/phage type combination was $S$. Enteritidis phagetype 1. Of the isolates $81.6 \%$ were susceptible to amoxicillin/clavulanic acid; $65.2 \%$ to ampicilin; $99.9 \%$ to ciprofloxacin; $93.4 \%$ to trimethoprim-sulphametoxazole; and $99.8 \%$ to cefotaxime. Molecular methods could be useful to complete epidemiologic studies since $25 \%$ of our isolates showed the same serotype/phage type combination. In our health district antimicrobial resistance in Salmonella is not an important problem.

Key words: Salmonella, serotyping, serotypes, phage types, antimicrobial resistance.

Palabras clave: Salmonella, serotipificación, serotipos, fagotipos, resistencia antimicrobiana.

\section{Introducción}

$\mathrm{L}$ a salmonelosis es un importante problema de salud pública a nivel mundial ${ }^{1}$. En España, Salmonella sp representa una de las principales causas de gastroenteritis infecciosa junto con Campylobacter sp y rotavirus ${ }^{2,3}$.

Aunque la infección intestinal, que se transmite al hombre a través de alimentos contaminados, es la forma clínica más importante, Salmonella sp también puede causar otros procesos tales como bacteriemias ${ }^{4,5}$, artritis ${ }^{6}$, meningitis ${ }^{7}$ y abscesos de órganos y tejidos ${ }^{8}$, entre otros.

Comprender la epidemiología de la salmonelosis resulta fundamental para conseguir el control sanitario de esta bacteria?. La serotipificación es el método de elección para realizar la tipificación de las cepas ${ }^{10}$. Conocer los serotipos de Salmonella sp y cómo se distribuyen en una zona geográfica determinada permite detectar brotes y distinguirlos de los casos esporádicos, identificar fuentes de infección y averiguar si existe asociación entre los diferentes serotipos y la resistencia a los antimicrobianos ${ }^{11}$.

Aunque se han descrito más de 2.500 serotipos de Salmonella $\mathrm{sp}^{12}$, los aislados de muestras clínicas pertenecen mayoritariamente a Salmonella Enteritidis y Salmonella Typhimurium ${ }^{13}$. Por esto se hace necesaria la utilización de técnicas complementarias como la fagotipificación que puede diferenciar cepas pertenecientes a un mismo serotipo $^{13}$. Esta técnica de caracterización fenotípica permite ampliar la información necesaria para establecer posibles relaciones epidemiológicas.

Habitualmente la salmonelosis cursa como un proceso autolimitado, pero también existen casos graves que pueden requerir hospitalización y en los que la instauración de un tratamiento antimicrobiano adecuado es primordial para la recuperación del paciente ${ }^{14}$. Algunos autores describen que la resistencia a los antimicrobianos considerados clásicamente de elección para el tratamiento está incrementándose ${ }^{15}$. Además, la proporción de aislados de Salmonella sp con susceptibilidad disminuida a fluoroquinolonas ha aumentado en muchos países, especialmente en los del sudeste asiático ${ }^{16}$.

El objetivo de este estudio es conocer la frecuencia y evolución de los distintos serotipos y fagotipos de Salmonella sp encontrados en el departamento de salud 02 de la provincia de Castellón, España, y su susceptibilidad a antimicrobianos.

\section{Método}

Estudio retrospectivo. Se recogieron tanto datos demográficos (edad, nacionalidad, procedencia ambulatoria 
u hospitalaria) como microbiológicos (tipo de muestra, mes y año de obtención de la cepa) de todos los casos con aislamiento de Salmonella sp en alguna muestra clínica, entre enero de 2000 y diciembre de 2006. Se incluyó sólo la primera cepa de cada episodio, independientemente del tipo de muestra en que se aislara.

La serotipificación se realizó en el Hospital General de Castellón por aglutinación sobre portaobjeto según el esquema de Kauffmann-White ${ }^{17}$. Se dispuso de los antisueros polivalentes (OMA y OMB), monovalentes para antígenos somáticos $(4,5,6,7,8,9$ y Vi) y flagelares (i,m,d,z10 y r) (Bio-Rad). Con ellos fue posible identificar los serotipos Salmonella Typhimurium, Salmonella Enteritidis, Salmonella Typhi, Salmonella Hadar y Salmonella Virchow. El resto de las cepas se caracterizó sólo a nivel de serogrupo B, C y D o como Salmonella sp.

Todos los aislados se remitieron al Laboratorio de Enterobacterias del Instituto de Salud Carlos III de Majadahonda (Centro Nacional de Referencia) para su completa serotipificación y para fagotipificar los serotipos que tenían un esquema reconocido internacionalmente: Typhimurium, Enteritidis, Typhi, Hadar y Virchow ${ }^{13}$.

El estudio de susceptibilidad se realizó en el año 2000 mediante el sistema de microdilución en placa MicroScan $\AA$ (Dade Behring) y desde el año 2001, por el sistema Vitek-2® (bioMérieux); en ambos casos, siguiendo las instrucciones de los fabricantes. Los antimicrobianos probados fueron amoxicilina/ác. clavulánico, ampicilina, cefotaxima, ciprofloxacina, norfloxacina, ofloxacina y cotrimoxazol. La interpretación de los resultados de las concentraciones inhibitorias mínimas (CIMs) se realizó de acuerdo con los criterios validados por el Clinical and Laboratory Standards Institute (CLSI).

Para valorar si había diferencias estadísticamente significativas de edad (proporciones de niños/adultos) en los grupos de pacientes hospitalizados y no hospitalizados se usó el test de $\chi^{2}$ con dos grados de libertad. Para comprobar si hubo cambios en la proporción de serotipos identificados cada año respecto al conjunto del periodo, se usó la prueba de $\chi^{2}$ de bondad de ajuste, comparando las proporciones observadas en cada uno de los años con la del conjunto del periodo, una comparación para cada año. Se distinguieron tres grupos: serotipo Enteritidis, serotipo Typhimurium y resto de serotipos. Se empleó regresión lineal simple, tomando como variable dependiente el número de cepas aisladas cada año y como variable independiente el año, para estimar si había tendencia en la evolución temporal de las cepas incluidas en el estudio. Se utilizaron los programas Epi Info v6.04 y SPSS v14.00 para realizar los cálculos, considerándose diferencia estadísticamente significativa cuando el valor $\mathrm{p}$ obtenido fuera inferior a 0,05 .

\section{Resultados}

El número total de pacientes fue de 1.505, de los cuales $54 \%$ eran hombres y $46 \%$ mujeres. Los pacientes con edad conocida fueron 1.298, siendo la media de edad de 14,4 años. Los grupos etarios donde más frecuentemente se aisló Salmonella fueron: bajo 5 años de edad (49,5\%), de 5 a 14 años $(17,8 \%)$ y de 25 a 34 años $(8 \%)$. Si se consideran sólo los pacientes en edad pediátrica (0-14 años), 29,3\% tenían menos de 2 años de edad.

Respecto a la nacionalidad, $6,6 \%$ de los pacientes eran extranjeros. Según procedencia, 67,4\% eran pacientes ambulatorios y $32,6 \%$ hospitalizados. Dentro de estos últimos, 50,5\% fueron ingresados en servicios pediátricos, $24,9 \%$ en la Unidad de Corta Estancia y $8,5 \%$ en Medicina Interna. La diferencia entre el grupo de niños hospitalizados (248/877) y el de adultos hospitalizados $(243 / 628)$ fue estadísticamente significativa $(\mathrm{p}<0,0001)$.

Los grupos de edad con mayor porcentaje de hospitalizados fueron aquellos sobre 65 años de edad (60\%), entre 45 y 54 años $(57,7 \%)$ y de 55 a 64 (50\%). El grupo bajo 5 años de edad presentó el menor porcentaje de hospitalizados $(23,5 \%)$, siendo éste muy similar cuando se estudiaron sólo los pacientes bajo 2 años de edad (26,6\%).

Un $98,1 \%$ de las salmonelas se aislaron en muestras de heces, $1,3 \%$ en sangre, $0,3 \%$ en orina, $0,1 \%$ en líquido pleural, $0,1 \%$ en líquido biliar y $0,1 \%$ en absceso intraabdominal.

La distribución por año de los casos fue: $14,6 \%$ en el año $2000 ; 12,1 \%$ en el 2001; $15,1 \%$ el año $2002 ; 16,5 \%$ en el $2003 ; 15,5 \%$ en el $2004 ; 14,9 \%$ en el 2005 y $11,2 \%$ el año 2006.

Los meses en los que más se aisló Salmonella sp fueron agosto $(13,1 \%)$, julio $(12 \%)$, septiembre $(10,8 \%)$ y junio $(10,1 \%)$.

En el periodo del estudio se identificaron 49 serotipos diferentes de Salmonella sp, siendo los más frecuentes: Enteritidis (51,7\%), Typhimurium (28,4\%), Hadar (5,2\%), serotipo 4,5,12:i:- (1,7\%), Rissen (1,4\%), Ohio (1,3\%) y Virchow (1,3\%). El resto de serotipos representó un porcentaje menor de 1\% cada uno. Enteritidis fue el serotipo predominante en todos los años excepto en el año 2005, en el que Typhimurium ocupó el primer lugar. El número de cepas de Salmonella Hadar descendió paulatinamente hasta el año 2004; sin embargo, en el 2005 se encontró el mayor número de aislados de este serotipo. En la Tabla 1 se observa la distribución de todos los serotipos por año de estudio.

La comparación de las proporciones de los serotipos considerados en cada uno de los años con la de todo el periodo (51,7\% Salmonella Enteritidis; 28,4\% Salmonella Typhimurium, y 19,9\% el resto) fue estadísticamente significativa sólo para los años 2003 ( $\mathrm{p}<0,001), 2005$ $(p<0,001)$ y $2006(p=0,006)$. No se observó tendencia 


\begin{tabular}{|c|c|c|c|c|c|c|c|c|c|}
\hline \multirow{2}{*}{ Tipo } & \multirow{2}{*}{$\begin{array}{c}2000 \\
n\end{array}$} & \multirow{2}{*}{$\begin{array}{c}2001 \\
n\end{array}$} & \multirow{2}{*}{$\begin{array}{c}2002 \\
n\end{array}$} & \multirow{2}{*}{$\begin{array}{c}2003 \\
n\end{array}$} & \multirow{2}{*}{$\begin{array}{c}2004 \\
n\end{array}$} & \multirow{2}{*}{$\begin{array}{c}2005 \\
n\end{array}$} & \multirow{2}{*}{$\begin{array}{c}2006 \\
n\end{array}$} & \multicolumn{2}{|c|}{ Total } \\
\hline & & & & & & & & $\mathbf{n}$ & (\%) \\
\hline Enteritidis & 117 & 91 & 125 & 159 & 113 & 73 & 100 & 778 & $(51,69)$ \\
\hline Typhimurium & 55 & 49 & 69 & 48 & 66 & 88 & 52 & 427 & $(28,37)$ \\
\hline Hadar & 15 & 9 & 5 & 5 & 3 & 36 & 5 & 78 & $(5,18)$ \\
\hline 4,5,12: i:- & 6 & 4 & 3 & 3 & 6 & 4 & & 26 & $(1,73)$ \\
\hline Rissen & 2 & 1 & 4 & 4 & 3 & 6 & 1 & 21 & $(1,39)$ \\
\hline Ohio & 5 & 7 & 4 & 3 & 1 & & & 20 & $(1,33)$ \\
\hline Virchow & 2 & 7 & 2 & 2 & 1 & 4 & 1 & 19 & $(1,26)$ \\
\hline Infantis & 2 & 1 & 4 & 2 & 2 & 1 & & 12 & $(0,80)$ \\
\hline Mbandaka & & & 1 & & 9 & 1 & & 11 & $(0,73)$ \\
\hline Bredeney & 1 & 2 & 2 & 3 & 1 & 1 & & 10 & $(0,66)$ \\
\hline 4,12: i:- & & & & 4 & 3 & 2 & & 9 & $(0,60)$ \\
\hline Brandenburg & 3 & & 1 & 2 & 2 & 1 & & 9 & $(0,60)$ \\
\hline Derby & 2 & 1 & 1 & 1 & 2 & & 1 & 8 & $(0,53)$ \\
\hline Newport & & & 2 & 2 & 4 & & & 8 & $(0,53)$ \\
\hline Mikawasima & 1 & 1 & 1 & 1 & 2 & & 1 & 7 & $(0,46)$ \\
\hline Montevideo & 2 & & & & 1 & 2 & 1 & 6 & $(0,40)$ \\
\hline Saintpaul & & & & 1 & 3 & & & 4 & $(0,28)$ \\
\hline London & & & & 2 & 2 & & & 4 & $(0,27)$ \\
\hline Pomona & & & & & & 1 & 3 & 4 & $(0,27)$ \\
\hline Anatum & 1 & & & & 2 & & & 3 & $(0,20)$ \\
\hline Grumpensis & & 3 & & & & & & 3 & $(0,20)$ \\
\hline Heidelberg & 1 & 1 & & 1 & & & & 3 & $(0,20)$ \\
\hline Livingstone & & 1 & & 1 & & & 1 & 3 & $(0,20)$ \\
\hline Panama & 1 & 1 & & 1 & & & & 3 & $(0,20)$ \\
\hline Wien & 1 & & 1 & & & & 1 & 3 & $(0,20)$ \\
\hline Indiana & & & & & 1 & 1 & & 2 & $(0,14)$ \\
\hline Paratyphi B & 2 & & & & & & & 2 & $(0,14)$ \\
\hline Otros* & 1 & 3 & 3 & 3 & 7 & 3 & 2 & 22 & $(1,54)$ \\
\hline Total & 220 & 182 & 228 & 248 & 234 & 224 & 169 & 1.505 & \\
\hline
\end{tabular}

lineal con significación estadística en la evolución temporal de las cepas incluidas en el estudio, ni aumento o disminución a lo largo de todo el período (Tabla 1).

En cuanto a los fagotipos, los más frecuentes de Salmonella Enteritidis fueron 1, 4, 6, 6a y 21 (Tabla 2); de Salmonella Typhimurium, 104b, U302, 193, 104 y
208 (Tabla 3); de Salmonella Hadar, 2, 1, 11, 14 y 29; del serotipo 4,5,12:i:-, U302, 193, 104, 104b y 120 y de Salmonella Virchow, los fagotipos 8, 17 y 19.

Al estudiar conjuntamente serotipos y fagotipos las combinaciones más halladas fueron: Salmonella Enteritidis fagotipo 1 (28,7\%), Salmonella Typhimurium 


\begin{tabular}{|c|c|c|c|c|c|c|c|c|c|}
\hline \multirow{2}{*}{ Fagotipo } & \multirow{2}{*}{$\begin{array}{c}2000 \\
n\end{array}$} & \multirow{2}{*}{$\begin{array}{c}2001 \\
n\end{array}$} & \multirow{2}{*}{$\begin{array}{c}2002 \\
n\end{array}$} & \multirow{2}{*}{$\begin{array}{c}2003 \\
n\end{array}$} & \multirow{2}{*}{$\begin{array}{c}2004 \\
n\end{array}$} & \multirow{2}{*}{$\begin{array}{c}2005 \\
\text { n }\end{array}$} & \multirow{2}{*}{$\begin{array}{c}2006 \\
n\end{array}$} & \multicolumn{2}{|c|}{ Total } \\
\hline & & & & & & & & $\mathbf{n}$ & (\%) \\
\hline 1 & 73 & 34 & 86 & 73 & 60 & 44 & 62 & 432 & $(55,53)$ \\
\hline 4 & 17 & 19 & 8 & 11 & 2 & & 1 & 58 & $(7,46)$ \\
\hline 6 & 3 & 3 & 3 & 19 & 13 & 5 & 5 & 51 & $(6,56)$ \\
\hline $6 \mathrm{~A}$ & 7 & 6 & 6 & 12 & 4 & & 2 & 37 & $(4,76)$ \\
\hline 21 & 2 & & 4 & 9 & 6 & 7 & 7 & 35 & $(4,50)$ \\
\hline $14 \mathrm{~B}$ & & & 1 & 5 & 13 & 3 & 5 & 27 & $(3,47)$ \\
\hline 3 & & 2 & 6 & 3 & 1 & & 3 & 15 & $(1,93)$ \\
\hline $5 C$ & & 13 & & & & & & 13 & $(1,67)$ \\
\hline 8 & 1 & 2 & 1 & 5 & 2 & 2 & & 13 & $(1,67)$ \\
\hline 41 & 6 & 4 & & & & & & 10 & $(1,30)$ \\
\hline $4 \mathrm{~B}$ & & 1 & & & & & 3 & 4 & $(0,52)$ \\
\hline $5 \mathrm{~A}$ & 1 & & 2 & 1 & & & & 4 & $(0,52)$ \\
\hline $21 C$ & & & 2 & & 1 & & & 3 & $(0,39)$ \\
\hline 1C & & & & & & & 2 & 2 & $(0,26)$ \\
\hline 2 & & & & & 1 & 1 & & 2 & $(0,26)$ \\
\hline $13 \mathrm{~A}$ & 1 & & 1 & & & & & 2 & $(0,26)$ \\
\hline 31 & & & & 1 & 1 & & & 2 & $(0,26)$ \\
\hline Otros* & 4 & 2 & & 2 & 1 & & 1 & 10 & $(1,3)$ \\
\hline $\mathrm{PNR}^{* *}$ & 2 & & 3 & 14 & 6 & 9 & 6 & 40 & $(5,14)$ \\
\hline$N T^{* * *}$ & & 5 & 2 & & & & 1 & 8 & $(1,03)$ \\
\hline Sin fagotipo & & & & 4 & 2 & 2 & 2 & 10 & $(1,30)$ \\
\hline Total & 117 & 91 & 125 & 159 & 113 & 73 & 100 & 778 & \\
\hline $\begin{array}{l}{ }^{*} \text { Otros (con } \\
\text { ** } \\
{ }^{*} \text { PNR: Patrć } \\
{ }^{* *} \text { NT: No } t\end{array}$ & $\begin{array}{l}\text { cada u } \\
\text { onocide }\end{array}$ & $5 B, 9 A$ & $21 \mathrm{~A}, 2$ & 37,44 & & & & & \\
\hline
\end{tabular}

fagotipo 104b (4,2\%), Salmonella Enteritidis fagotipo 4 (3,8\%), Salmonella Typhimurium fagotipo U302 (3,8\%) y Salmonella Enteritidis fagotipo 6 (3,4\% - 51/1.505) (Tabla 3).

Los porcentajes de susceptibilidad global encontrados frente a cada antimicrobiano fueron: amoxicilina/ác. clavulánico, 81,6\%; ampicilina, 65,2\%; ciprofloxacina 99,9\%; cotrimoxazol, 93,4\%; y cefotaxima, 99,8\% (en la Tabla 4 se describe la susceptibilidad por año de estudio y antimicrobiano). Los porcentajes de resistencia y de sensibilidad intermedia fueron respectivamente: amoxicilina/ác. clavulánico, 2,5\% y 15,9\%; ampicilina, $34,7 \%$ y $0,1 \%$; ciprofloxacina $0 \%$ y $0,1 \%$; cotrimoxazol, $6,6 \%$ y $0 \%$; y cefotaxima, $0 \%$ y $0,2 \%$. El año con mayor porcentaje de cepas con resistencia y/o sensibilidad intermedia a amoxicilina/ác. clavulánico, ampicilina, y cotrimoxazol fue el 2005.

Presentaron resistencia y/o sensibilidad intermedia a uno o más de los antimicrobianos estudiados $86,2 \%$ de Salmonella Typhimurium, 80,8\% de Salmonella Hadar, $69,2 \%$ del serotipo 4,5,12:i:-, 21\% de Salmonella Virchow, y 11,9\% de Salmonella Enteritidis.

De todas las cepas de Salmonella sp aisladas sólo hubo tres cepas con sensibilidad intermedia a cefotaxima: una serotipo Mbandaka, otra Typhimurium y otra Virchow; y una cepa de Salmonella Enteritidis con sensibilidad intermedia a ciprofloxacina. No se estudió el mecanismo subyacente. 


\begin{tabular}{|c|c|c|c|c|c|c|c|c|c|}
\hline Fagotipo & $\begin{array}{c}2000 \\
n\end{array}$ & $\begin{array}{c}2001 \\
n\end{array}$ & $\begin{array}{c}2002 \\
n\end{array}$ & $\begin{array}{c}2003 \\
n\end{array}$ & $\begin{array}{c}2004 \\
n\end{array}$ & $\begin{array}{c}2005 \\
n\end{array}$ & $\begin{array}{c}2006 \\
n\end{array}$ & $n$ & $\begin{array}{l}\text { otal } \\
\text { (\%) }\end{array}$ \\
\hline $104 B$ & 4 & 6 & 16 & 9 & 10 & 15 & 4 & 64 & $(14,99)$ \\
\hline U302 & 2 & 1 & 14 & 11 & 9 & 13 & 7 & 57 & $(13,35)$ \\
\hline 193 & 1 & 2 & 11 & 3 & 10 & 4 & 8 & 39 & $(9,13)$ \\
\hline 104 & 11 & 4 & 7 & & 3 & 3 & 2 & 30 & $(7,02)$ \\
\hline 208 & 2 & 2 & 1 & 1 & 3 & 1 & & 10 & $(2,34)$ \\
\hline 120 & & & 1 & & & 5 & 3 & 9 & $(2,11)$ \\
\hline 195 & 1 & & 1 & 3 & 2 & & & 7 & $(1,64)$ \\
\hline 68 & & 1 & 4 & 1 & & & & 6 & $(1,40)$ \\
\hline 4 & & 2 & & & & & & 2 & $(0,47)$ \\
\hline 12 & & & & & 2 & & & 2 & $(0,47)$ \\
\hline 41 & & 1 & & & & & 1 & 2 & $(0,47)$ \\
\hline 137 & & & & & & 1 & 1 & 2 & $(0,47)$ \\
\hline Otros* & & 4 & 1 & 5 & 1 & & 1 & 12 & $(2,76)$ \\
\hline PNR $^{* *}$ & 2 & 1 & 4 & 4 & 10 & 15 & 4 & 40 & $(9,37)$ \\
\hline$N T^{* * *}$ & 20 & 19 & 7 & 11 & 14 & 28 & 20 & 119 & $(27,87)$ \\
\hline sin fagotipo & 12 & 6 & 2 & & 2 & 3 & 1 & 26 & $(6,09)$ \\
\hline Total & 55 & 49 & 69 & 48 & 66 & 88 & 52 & 427 & \\
\hline \multicolumn{10}{|c|}{$\begin{array}{l}\text { *Otros (con una cepa cada uno): 3, 18, 2, 7, 8, 10, 52a, } 56 \text { variante, 170, 179, 185, U283. } \\
\text { ** PNR: Patrón no reconocido. } \\
\text { *** NT: No tipificable. }\end{array}$} \\
\hline
\end{tabular}

\section{Tabla 4. Distribución anual de la susceptibilidad de Salmonella sp a los antimicrobianos estudiados}

\begin{tabular}{|c|c|c|c|c|c|c|c|c|c|c|c|c|c|c|c|c|}
\hline & \multicolumn{2}{|c|}{2000} & \multicolumn{2}{|c|}{2001} & \multicolumn{2}{|c|}{2002} & \multicolumn{2}{|c|}{2003} & \multicolumn{2}{|c|}{2004} & \multicolumn{2}{|c|}{2005} & \multicolumn{2}{|c|}{2006} & \multicolumn{2}{|c|}{ Global } \\
\hline & $\mathbf{n}$ & $\%$ & $\mathbf{n}$ & $\%$ & $\mathbf{n}$ & $\%$ & $\mathbf{n}$ & $\%$ & $\mathbf{n}$ & $\%$ & $\mathbf{n}$ & $\%$ & $\mathbf{n}$ & $\%$ & $\mathbf{n}$ & $\%$ \\
\hline AMC & 186 & $(84,5)$ & 151 & (83) & 204 & $(89,5)$ & 220 & $(88,7)$ & 191 & $(81,6)$ & 131 & $(58,5)$ & 145 & $(85,8)$ & 1.228 & $(81,6)$ \\
\hline Ampicilina & 144 & $(65,4)$ & 124 & $(68,1)$ & 157 & $(68,8)$ & 174 & $(70,2)$ & 158 & $(67,5)$ & 104 & $(46,4)$ & 120 & (71) & 981 & $(65,2)$ \\
\hline Ciprofloxacina & 220 & $(100)$ & 182 & $(100)$ & 228 & (100) & 248 & $(100)$ & 233 & $(99,6)$ & 224 & $(100)$ & 169 & $(100)$ & 1.504 & $(99,9)$ \\
\hline Cotrimoxazol & 208 & $(94,5)$ & 169 & $(92,8)$ & 216 & $(94,7)$ & 233 & $(93,9)$ & 217 & $(92,7)$ & 201 & $(89,7)$ & 161 & $(95,3)$ & 1.405 & $(93,3)$ \\
\hline Cefotaxima & 219 & $(99,5)$ & 182 & (100) & 227 & $(99,6)$ & 248 & $(100)$ & 234 & $(100)$ & 223 & $(99,5)$ & 169 & $(100)$ & 1.502 & $(99,8)$ \\
\hline Total de cepas & 220 & & 182 & & 228 & & 248 & & 234 & & 224 & & 169 & & 1.505 & \\
\hline $\begin{array}{l}\text { AMC: Amoxicil } \\
\text { R: resistente. I: } \\
\text { N: no de cepas } \\
\%: \text { porcentaje }\end{array}$ & $\begin{array}{l}\text { bilida } \\
\text { bles. } \\
\text { asibili }\end{array}$ & $\begin{array}{l}\text { ulánico. } \\
\text { d interme } \\
\text { dad. }\end{array}$ & & & & & & & & & & & & & & \\
\hline
\end{tabular}




\section{Discusión}

Este trabajo refleja el perfil epidemiológico de Salmonella sp en nuestro departamento de salud durante los siete años de estudio, y aporta una aproximación general de la distribución de serotipos, fagotipos y el nivel de resistencia de los aislados clínicos de Salmonella sp de nuestro país.

En los laboratorios clínicos resulta complicado realizar de rutina la serotipificación completa y aún más la fagotipificación de Salmonella sp. Aunque existe la posibilidad de remitir las cepas aisladas a un centro de referencia, esto supone una carga de trabajo adicional que no siempre se puede llevar a cabo. Nuestro hospital envía sistemáticamente una cepa de cada episodio de salmonelosis al Laboratorio Nacional de Referencia de Salmonella y Shigella para su sero y fagotipificación, información que es utilizada para la investigación de brotes.

En nuestro estudio, la distribución por sexo de los pacientes fue muy similar a la encontrada en otras series $^{13,18}$. El grupo de niños bajo 5 años de edad concentró el mayor número de aislados de cepas de Salmonella $\mathrm{sp}$, hecho muy semejante a lo descrito por Gil-Setas y cols ${ }^{18}$ y Juncosa y cols ${ }^{19}$.

Los pacientes con salmonelosis que tienen mayor riesgo de complicaciones $\mathrm{y}$, por lo tanto, requieren mayor número de ingresos hospitalarios, son los lactantes y los ancianos ${ }^{20}$. En nuestro estudio el grupo de edad con mayor porcentaje de hospitalizaciones fue el sobre 65 años de edad (60\%), lo que concuerda con la literatura médica. Sin embargo, los lactantes y niños bajo 5 años de edad presentaron el menor porcentaje de hospitalizados $(23,52 \%)$.

Los meses con mayor proporción de aislados a nivel global fueron los de verano, dato que coincide con el de otros autores ${ }^{18,19}$. Cabe destacar que en julio de 2002 hubo un número anormalmente alto de aislados (60) debido a un brote por Salmonella Enteritidis fagotipo 1 .

Coincidimos con otros autores españoles ${ }^{18,21-23}$ y europeos $^{24}$ en que el serotipo predominante es Enteritidis. Esto difiere de lo que ocurre en otros países como E.U.A. ${ }^{11}$, México $^{25}$ o Taiwan ${ }^{26}$, donde el serotipo más aislado es Typhimurium.

En orden de frecuencia, Salmonella Hadar (5,18\%) ocupa el tercer lugar, al igual que en otras series ${ }^{16,21,23}$. Nuestra prevalencia es mayor que la que la publicada en distintas zonas de nuestro país. En Córdoba, De Carlos y cols $^{10}$ comunican 3,5\%; en Pontevedra, Bellver y cols ${ }^{21}$ 2,3\%; y en el Principado de Asturias, González y cols ${ }^{22}$ $4,5 \%$. Esto es debido, posiblemente, a los distintos periodos abarcados en los diferentes estudios y a que hubo un brote de salmonelosis en nuestra comunidad en el año 2005 por ingestión de carne de pollo envasada, contaminada con Salmonella Hadar.

A pesar de que algunos autores consideran el serotipo 4,5,12:i:- como una variante monofásica de Salmonella Typhimurium $^{13}$, y se emplee su mismo esquema de fagotipificación, nosotros lo hemos considerado como un serotipo independiente, al igual que Echeita y cols ${ }^{13}$.

Comparando los distintos estudios publicados, podemos apreciar que la frecuencia de algunos serotipos menos habituales es muy variable, lo que puede deberse a la aparición de brotes. Por ejemplo, en nuestro caso, un brote en el año 2004 por Salmonella Mbandaka hizo que el número global de casos aumentara, de forma que ocupa el $9^{\circ}$ lugar mientras que en otros trabajos españoles es menos frecuente ${ }^{10,16}$.

La distribución de los fagotipos más frecuentes por serotipo es muy similar a la descrita por otros autores ${ }^{10,14,16}$. No obstante, la frecuencia de fagotipo 1 en Salmonella Enteritidis en nuestra área $(55,53 \%)$ es mayor que la comunicada por el estudio nacional de Echeita y cols $(35,92 \%)^{16}$.

Las técnicas de sero/fagotipificación no son suficientemente discriminativas para estudios epidemiológicos de cepas cuando se caracterizan tipos muy frecuentes. En nuestro caso, más de $25 \%$ de todos los aislados presentó la misma combinación de serotipo y fagotipo: Salmonella Enteritidis fagotipo 1. Debido a esto, para asegurar la identidad de las cepas sería necesario recurrir a métodos moleculares como la electroforesis de campo pulsado (PFGE), la reacción de polimerasa en cadena (RPC), la amplificación randómica de ADN polimórfico (RAPD PCR) y la ribotipificación ${ }^{27}$; técnicas, todas ellas, complejas y de coste elevado.

Aunque hay autores que comunican el aumento de la resistencia de Salmonella sp frente a los antimicrobianos considerados clásicamente de elección para su tratamiento ${ }^{15}$, e incluso hay quien ya describe alguna resistencia a ciprofloxacina ${ }^{18,28}$, nosotros no hemos observado este incremento durante los años del estudio. Sin embargo, se aprecia que el año 2005 presentó el menor porcentaje de cepas sensibles a amoxicilina/ác. clavulánico, ampicilina y cotrimoxazol, seguramente porque fue el año con el mayor porcentaje de cepas de Salmonella Typhimurium, que es el serotipo con mayor número de cepas resistentes.

La resistencia a ampicilina fue de $34,68 \%$, porcentaje muy similar al descrito por otros autores ${ }^{18,29}$, aunque Bellver y cols ${ }^{21}$ presenten un porcentaje mayor (39\%) y Soler P y cols ${ }^{14}$ uno menor $(28,4 \%)$.

La resistencia a cotrimoxazol fue de 6,64\% como la descrita por Soler P. y cols ${ }^{14}(6 \%)$. Otros estudios ${ }^{21,29}$ describen aún menor resistencia $(3,5 \%)$. Por esta razón, pensamos que este antimicrobiano todavía mantiene una buena actividad en nuestro medio. 
No se encontró cepa alguna resistente a cefotaxima ni a ciprofloxacina (aunque no se evaluó la susceptibilidad disminuida a quinolonas en las cepas de las muestras extra-intestinales, por lo que desconocemos si alguno de nuestros aislados presenta esta característica).

De todos los serotipos, Typhimurium es el que presenta un mayor porcentaje de cepas con resistencia o sensibilidad intermedia a los antimicrobianos estudiados, lo que concuerda con la literatura médica revisada $^{18,21,29}$.

Consideramos que la información presentada respecto a Salmonella sp es amplia y que ayuda al control de la salmonelosis en nuestra área, aunque sería de utilidad disponer de técnicas moleculares que completaran los estudios epidemiológicos. Este trabajo contribuye a aumentar el conocimiento epidemiológico de Salmonella sp en España y muestra que en nuestro departamento la resistencia antimicrobiana de esta bacteria no supone un problema tan relevante como el comunicado por otros autores.

\section{Resumen}

Estudio retrospectivo de los serotipos, fagotipos y la resistencia antimicrobiana de los aislados de Salmonella sp en el departamento de salud 02 de Castellón, España (2000-2006). La serotipificación se realizó usando antisueros comerciales y el estudio de susceptibilidad mediante sistemas automatizados. Todas las cepas se enviaron al Centro Nacional de Referencia para confirmar el serotipo y realizar la fagotipificación. Se aislaron 1.505 cepas identificándose 49 serotipos diferentes, siendo el más frecuente Enteritidis. La combinación serotipo/fagotipo más hallada fue Salmonella Enteritidis fagotipo 1. Los porcentajes de susceptibilidad fueron: amoxicilina/ác. clavulánico, 81,6\%; ampicilina, 65,2\%; ciprofloxacina 99,9\%; cotrimoxazol, 93,4\%; y cefotaxima, 99,8\%. Serían necesarias técnicas moleculares que completasen los estudios epidemiológicos ( $25 \%$ de nuestros aislados presentaron la misma combinación serotipo/fagotipo). Las resistencias antimicrobianas no suponen en nuestro departamento un problema relevante.

\section{Referencias}

1.- Chalker R, Blaser M. A Review of human Salmonellosis III: magnitude of Salmonella infection in the United States. Rev Infect Dis 1988; 10: 111-24.

2.- Bellido J B, González J M, Galiano J V, Herrero C, Tirado M D, Arnedo A et al. Factores de riesgo de los casos esporádicos de diarrea por Campylobacter, Salmonella y rotavirus en niños preescolares. An Pediatr 2007; 66: 367-74.

3.- Bellido J B, Galiano J V, Tirado M D,

González J M, Safont L. Incidencia de casos esporádicos de las infecciones intestinales en Castellón. Rev Esp Salud Pública 2003; 77: 629-38.

4.- Barrio J, Castiella A, Von Wichman M A, Cosme A, López P, Arenas JI. Spontaneous bacteriemia due to Salmonella hadar in liver cirrosis with transjugular intrahepatic portosystemic shunt. Gastroenterol Hepatol 1999; 22: 79-81.

5.- $\quad$ Preceden T, Knezevic K, Brkic S, Jelesic Z. Salmonella bacteriemia. Med Pregl 2001; 54: 367-70.

6.- Suwannaroj S, Mootsikapun P, Vipulkorn K, Nanagara R. Salmonella group D septic arthritis and necrotizing fasciitis in a patient with rheumatoid arthritis and diabetes melllitus. J Clin Rheumatol 2001; 7: 83-5.

7.- $\quad$ Choudhury SA, Berthaud V, Weitkamp JH. Meningitis cause by Salmonella panama in infants. J Natl Med Assoc. 2006; 98: 219-22.

8.- Millar A S, Hunstad D A. Salmonella ovarian abscess in an adolescent. Pediatr Infect Dis J 2007 Jun; 26: 548-9.

9.- Desenclos JC, Vaillant V, De Valk H. Foodborne infections: do we need further data collection for public health acting and research. Rev Epidemiol Sante Publique 2002; 50: 67-79.

10.- De Carlos F, Solís F, Navarro F, Muñoz J, Tejero R, Ibarra A et al. Salmonella spp. Serotipos aislados en el Hospital Universitario Reina Sofía de Córdoba durante un período de 8 años (1993-2000). Enferm Infecc Microbiol Clin 2002; 20: 208-11.

11.- Vugia D J, Samuel M, Farley M M, Marcus R, Shiferaw B, Shalow $\mathrm{S}$ et al. Emerging Infectious Program FoodNet Working Group. Invasive Salmonella infections in the United States, FoodNet, 1996-1999: incidence, serotype distribution and outcome. Clin Infect Dis 2004; 38 Suppl 3: S 149-56.

12.- Popoff M Y, Bockemuhl J, Gheesling L L. Supplement 2001 (no. 45) to the Kauffmann White scheme. Res Microbiol 2003; 154:173-4.

13.- Echeita M A, Aladueña A M, Díez R, Arroyo M, Cerdán F, Gutiérrez R, et al. Distribución de los serotipos y fagotipos de Salmonella de origen humano aislados en España en 1997-2001. Enferm Infecc Microbiol Clin 2005; 23: 127-34.

14.- Soler P, González-Sanz R, Bleda M J, Hernández G, Echeita A, Usera M A. Antimicrobial resistance in non-typhoidal Salmonella from human sources, Spain, 2001-2003. J Antimicrob Chemother 2006; 58 : 310-4.

15.- Ramos J M, Alés J M, Cuenca-Estrella M,
Fernández-Roblas R, Soriano F. Changes in susceptibility of Salmonella Enteritidis, Salmonella Typhimurium, and Salmonella Virchow to six antimicrobial agents in a Spanish hospital, 1980-1994. Eur J Clin Microbiol Infect Dis 1996; 15: 85-8.

16.- Hakanen A J, Kotilainen P, Pitkanen S, Huikko S, Siitonen A, Huovinen P. Reduction in fluoroquinolone susceptibility among nontyphoidal strains of Salmonella enterica isolated from Finnish patients. J Antimicrob Chemother 2006 Mar; 57: 569-72.

17.- Poppoff M Y. Antigenic formulas of the Salmonella serovars. $8^{\text {th }}$ revision. WHO-Collaborating Centre of Reference and Research on Salmonella. Paris: Institut Pasteur, 2001.

18.- Gil-Setas A, Mazón Ramos A, Martín Salas C, Urtiaga Domínguez M, Inza Elia ME. Salmonelosis no tifoidea en un área de salud de Navarra, España. Rev Esp Salud Pública 2002; 76: 49-56

19.- Juncosa T, Palacín E, Latorre C. Salmonelosis en un hospital materno-infantil de Barcelona durante 10 años (1992-2001). An Pediatr 2005; 63: 403-8

20.- Trevejo R T, Courtney J G, Starr M, Vugia D J. Epidemiology of salmonellosis in California, 1990-1999: morbidity, mortality, and hospitalization costs. Am J Epidemiol. 2003; 157: 48-57

21.- Bellver P, García M. Epidemiología de la salmonelosis no tifoidea en un hospital de Pontevedra (1994-1997). Enferm Infecc Microbiol Clin 2000; 18: 125-132.

22.- González-Hevia M A, Cruz Martín M C, Lobato M J, Gutiérrez F, Solano P, Álvarez- 
Riesgo J A. Salmonella y salmonelosis en el Principado de Asturias durante un periodo de 7 años (1990-1996). Enferm Infecc Microbiol Clin 1999; 17: 166-70.

23.- Sirvent E, Ruiz M, Rodríguez JC, Royo G. Serotipos y sensibilidad antibiótica del género Salmonella en Elche. Rev Esp Quimioter 2002; 15: 55-60.

24.- Czerwiński M. Salmonellosis in Poland in 2004 Przegl Epidemiol 2006; 60:429-40.

25.- Gutiérrez-Coqco L, Montiel-Vázquez E, Aguilera-Pérez P, González-Andrade MC. Serotipos de Salmonella identificados en los servicios de salud de México. Rev Salud Pública Mex 2000; 42: 490-5

26.- Lauderdale T L, Aarestrup F M, Chen P C, Lai J F, Wang H Y, Shiau Y R, et al. Multidrug resistance among different serotypes of clinical Salmonella isolates in Taiwan. Diagn Microbiol Infect Dis 2006; 55: 149-55.

27.- Silva J, Aravena C, Araya J, Colque-Navarro P, Kühne I, Möllby R. Fenotipos bioquímicos y fagotipos de cepas de Salmonella enteritidis aisladas en Antofagasta, 1997-2000. Rev Med
Chile 2003; 131: 837-45.

28.- Campo P, Gutiérrez A, Ladrón de Guevara C. Evolution of susceptibility of non typhi Salmonella in a Spanish hospital (1992-1994) and report of a Salmonella ser. Typhimurium isolate resistant to quinolones. Eur J Epidemiol 1997; 13: 239-41.

29.- López de Goicoechea M J, Echeverría M J, Berdonces P, Ayarza R. Estudio de sensibilidad de Salmonella spp no typhi en el Hospital de Galdakao (1992 a 1998). Rev Esp Quimioter 2001; 14: 51-4. 